\title{
Theoretical calculation of mid-infrared spectra from hypersonic non-ablative sphere
}

\author{
Wu Jie ${ }^{1,2}$, Yu Xilong ${ }^{1}$,Zhu Xijuan ${ }^{2}$,Ma Jing ${ }^{2}$, Mao Hongxia ${ }^{2}$ \\ 1.State Key Laboratory of High Temperature Gas Dynamics, Institute of Mechanics, Chinese \\ Academy of Sciences, Beijing, 100190, China \\ 2.Science and Technology on Optical Radiation Laboratory; Beijing 100854; China;
}

\begin{abstract}
Abstract: Hypersonic body moving in the atmosphere will suffer high temperature reacting flows which will emit complex radiation. Theoretical calculation was taken in this paper for a hypersonic non-ablative sphere. Hypersonic flow around the sphere was simulated using 9 species chemical kinetic and two temperature thermal non-equilibrium model. Based on this simulated flow field, the LOS method is used to solve radiative transfer and line-by-line model is used to calculate the spectrum from molecular and atoms in mid-infrared. The spectra from different components have been analyzed one by one. The calculation founds out that atom $\mathrm{N}$ and $\mathrm{O}$ diatomic molecule $\mathrm{NO}$ and bremsstrahlung will be important radiation source in this pure air hypersonic flow field. The radiation from hypersonic flow field has been analyzed in both high pressure environment and low pressure environment.
\end{abstract}

Keywords: hypersonic, mid-infrared, atom, diatomic molecule

\section{INTRODUCTION}

When a hypersonic body moving in the atmosphere, shock forms around the body and because of intense compression and viscous friction, air in the shock layer will be heated up to tens of thousands degrees. Upon such a high energy level, air molecule tend to be chemical reactive and inner vibrational because of more frequently molecular collision. Consequently, dissociation and ionization of the air happen, vibrational and electronic energy models of molecular are excited, and non-equilibrium radiation processes take place between different energy levels. Hence, the flow field

Corresponding author. Tel.: +86 15810253054; fax: +86 68387207

E-mail address: waterwu10@126.com ( Wu Jie )

Infrared Technology and Applications, and Robot Sensing and Advanced Control,

edited by Haimei Gong and Aiguo Song, Proc. of SPIE Vol. 10157, 1015727

(C) 2016 SPIE $\cdot$ CCC code: 0277-786X/16/\$18 · doi: 10.1117/12.2246778 
around a hypersonic body is high temperature plasma composed of different particles such as molecule, atoms and ions, which are in dynamic change.

In such high temperature condition, the radiation from sufficiently excited gas will mainly be in ultraviolet to visible bands, like Schumann-Range (B-X) system from $\mathrm{O}_{2}$ and $\gamma(\mathrm{A}-\mathrm{X})$ system from NO. The UV spectrum from high temperature gas has been calculated by NEQAIR ${ }^{[1]}$ and Specair ${ }^{[2]}$ quite well. The energy emissed by high temperature gas in min-infrared is not as much as in UV range, but mid-infrared range is important for detecting in atmosphere. So in this paper, the mid-infrared spectrum from hypersonic flow field was calculated. The theory calculation includes free-free transition, free-bound transition and bound-bound transition. Atom $\mathrm{N} \mathrm{O}$ and diatomic molecule NO are main emission source, because polyatomic molecular like CO2 are almost dissociated and homonuclear diatomic molecular cannot radiate in infrared.

\section{THEORY CALCULATION OF FLOW FIELD}

In order to obtain flow field solutions with thermochemical nonequilibrium around a sphere body, axisymmetric Navier-Stokes equations are calculated with CFD techniques. 9 species are included in high temperature air $\left(\mathrm{N}_{2}, \mathrm{O}_{2}, \mathrm{~N}, \mathrm{O}, \mathrm{NO}, \mathrm{NO}+, \mathrm{e}-\mathrm{CO}, \mathrm{CO}_{2}\right)$ and chemical reactions proceed at a finite rate, which employs the Arrhenius form to compute the production terms .

Two temperature model ${ }^{[3]}$ is employed to describe thermal non-equilibrium state of the flow, Landau-Teller model is employed to describe the energy exchange between the transitional-rotational and vibrational model. The relaxation time of the energy exchange is calculated with Millikan-White formula ${ }^{[4]}$. The grid for computation is seen in fig 1.

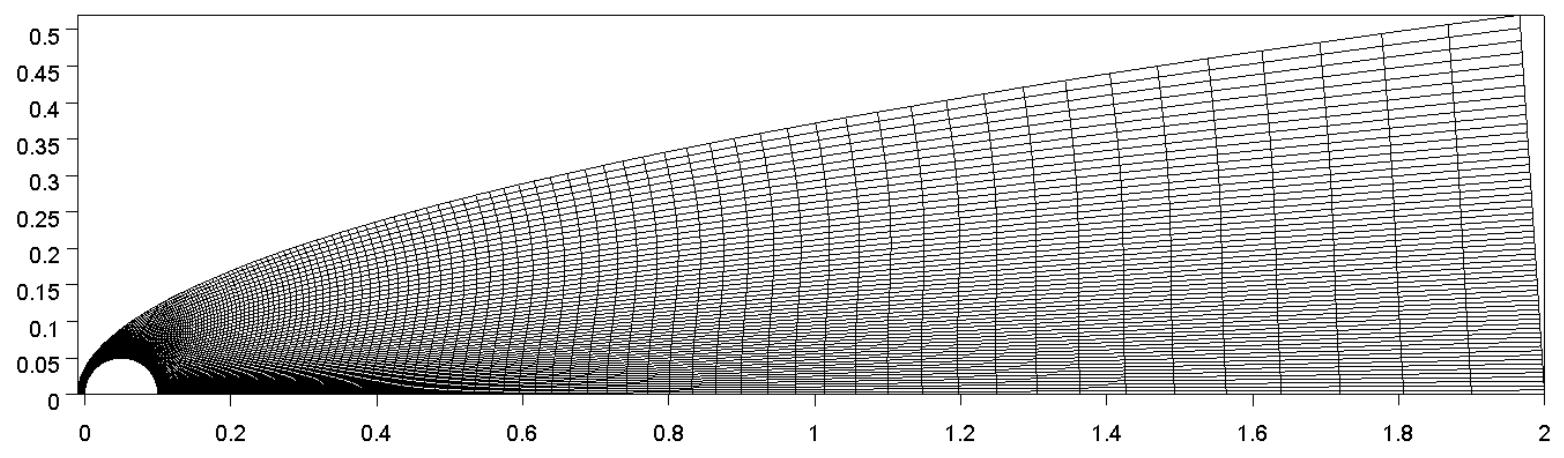

Figure 1 the grid for computation 


\section{THEORY CALCULATION OF SPECTRUM FROM GAS}

\section{1 basic theories}

The radiative transfer is solved by LOS method ${ }^{[5]}$ here. It means that scattering is ignored in this condition. For hypersonic flow field without ablating, there is nothing scattering mid-infrared light.

The equation of radiative transfer becomes one-dimensional as

$$
\frac{d I_{v}}{d x}=\kappa_{v}\left[B_{v}(T)-I_{v}\right]
$$

Where

$B_{v}=$ Plank black-body function, $\mathrm{W} / \mathrm{cm}^{2} \cdot \mathrm{sr}^{-} \cdot \mathrm{cm}^{-1}$

$\kappa_{v}=$ absorption coefficient, $\mathrm{cm}^{-1}$

$I_{v}=$ specific intensity, $\mathrm{W} / \mathrm{cm}^{2} \cdot \mathrm{sr}^{-} \mathrm{cm}^{-1}$

The CFD grid is used here to disperse one-dimensional ray, because CFD grid resolves the gradient of thermal states in flow field quiet well.

Absorption coefficient is the most important parameter calculated in this paper. The absorption coefficient is mainly dominated by atoms and diatomic molecular radiation and bremsstrahlung.

The emission from atom and molecule is bound-bound system; the spectrum is formed by many lines. The absorption coefficient of atom and molecule can be expressed by overlying of every line:

$$
\begin{aligned}
\kappa(v) & =\sum_{i} \kappa^{i}(v) \\
& =\sum_{i} S f\left(v-v_{0 i}\right) N
\end{aligned}
$$

Here, $\kappa^{i}(v)$ is the absorption coefficient of line i, $S$ is the integrated line intensity due to absorption, $f\left(v-v_{0 i}\right)$ is the function of line profile, $N$ is the number density, $v_{0 i}$ is the wave number of line $i$. The expression of integrated intensity is:

$$
E=\frac{N_{u} h v A_{u l}}{4 \pi}
$$

Here, $E$ is the integrated line intensity due to spontaneous emission, the relationship between $E$ and $S$ is fixed by Kirchhoff law. $N_{u}$ is the number of molecules in the upper state, $v$ is the frequency of 
line center, $A_{u l}$ is the Einstein coefficient. For atomic radiation calculation, the parameters above are from ASD (atomic spectra database) of NIST, some lines are correct by experiment data. For molecular calculation, the spectral parameters are from HITEMP 2010 database $^{[6]}$, some lines from $\mathrm{NO}$ overtone bands are correct by ourselves ${ }^{[7]}$ with ab initio theory.

In thermodynamic equilibrium condition, the upper state number density is given by Boltzmann distribution:

$$
N_{u}=\frac{g_{u} \exp \left(-E_{u} / k T\right)}{Q_{a}} N_{a}
$$

Here, $g_{u}$ is the multiplicity of upper state, $E_{u}$ is the energy of upper state, $Q_{a}$ is the partition function, $N_{a}$ is the number density of species. The partition functions for molecular from $300 \mathrm{~K}$ to 10000K are calculated using McDowell methods ${ }^{[8]}$.

For atmosphere in high altitude, the line profile must consider both collision broadening and Doppler broadening. The line shape is approximated by the Voigt profile ${ }^{[9]}$, which integrating Lorentz profile and Doppler profile. The expression is

$$
f\left(v-v_{0}\right)=\alpha_{D}^{-1} \pi^{-3 / 2} y \int_{-\infty}^{\infty} \frac{\exp \left(-t^{2}\right) d t}{y^{2}+\left[t-\left(v-v_{0}\right) / \alpha_{D}\right]^{2}}
$$

Where $y=\frac{\alpha_{L}}{\alpha_{D}}, \alpha_{L}$ and $\alpha_{D}$ are width of Lorentz profile and Doppler profile.

The bremsstrahlung is continuous radiation. For the speed about $5 \mathrm{~km} / \mathrm{s}$, the ionizing process in flow field is far from complete, so both of ions and neural must be taken into account for bremsstrahlung. The absorption coefficient of bremsstrahlung with ions is express by Kramers equation.

$$
\begin{aligned}
\kappa_{i} & =\frac{4}{3}\left(\frac{2 \pi}{3 k T_{e} m_{e}}\right)^{0.5} \frac{e^{6}}{\left(4 \pi \varepsilon_{0}\right)^{3} h m_{e} c v^{3}} Z^{2} n_{i} n_{e} \\
& =3.68 \times 10^{-2} \frac{Z^{2} n_{i} n_{e}}{\sqrt{T_{e}} v^{3}}
\end{aligned}
$$

Where $T_{e}$ is the electron temperature, $n_{i}$ the density of species i, $Z$ the charge of the ion, and $v$ the frequency.

The absorption coefficient of bremsstrahlung with neutral is defined as 


$$
\kappa_{i}=n_{e} n_{a i} Q_{a i}
$$

Where $n_{a i}$ is the density of neutral species i, $Q_{a i}$ the cross sections which are obtained from Kivel ${ }^{[10]}$.

\section{2 verification}

The plasma torch was used by Stanford University C. O. Laux et al ${ }^{[11]}$ to get high-temperature thermodynamic equilibrium air, and the spectrum from that air has been measured. The air injected into the torch contained approximately $330 \mathrm{ppm}$ of $\mathrm{CO} 2$ and $4.5 \mathrm{e}-3$ mole fraction of water vapor. The highest temperature of plasma plume is $7500 \mathrm{~K}$. The measured temperature profile was obtained from Abel-inverted profiles of some absolute $\mathrm{N}$ and $\mathrm{O}$ lines intensities. The concentrations of the different species used in the simulations are calculated by assuming chemical equilibrium at atmospheric pressure and at the measured temperatures. The temperature and mole fractions of the main species are plotted below:

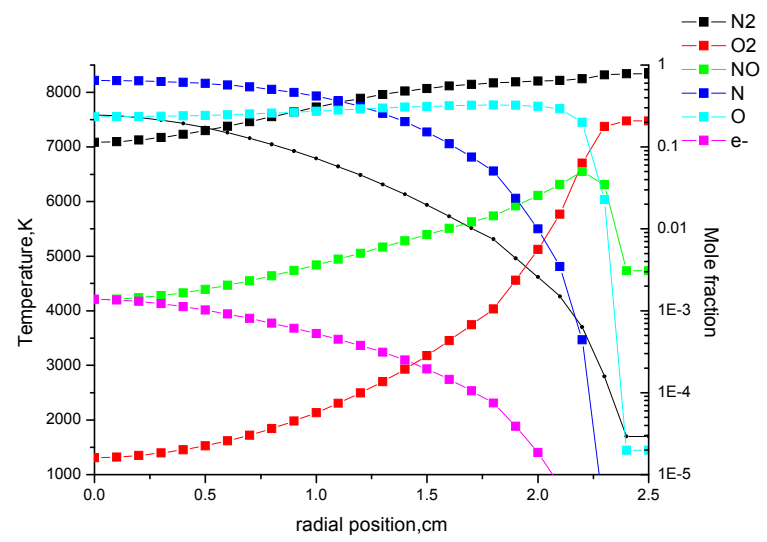

Figure 2 radial temperature and mole fraction profiles

In center of plasma plume, $\mathrm{N}_{2}$ and $\mathrm{O}_{2}$ are dissociated, and the mole fractions of $\mathrm{N}$ and $\mathrm{O}$ rise greatly. Because of ionization of atoms and molecular, there is some electron in plume. The mole fraction of NO can reach $10 \%$. Based on the radial temperature and mole fraction profiles, the spectrum of plume in mid-infrared is simulated by LOS method. 

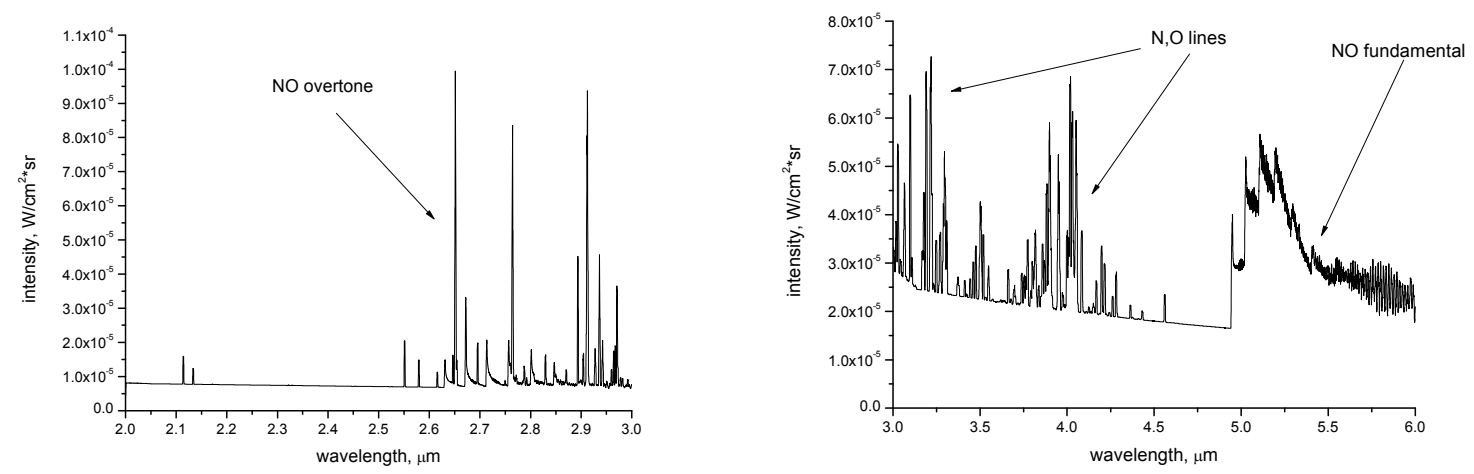

Figure 3 theory calculated spectrum from plasma plume

The measured and NEQAIR2-IR theory calculated spectra of plasma plume in 2-5.5 $\mu \mathrm{m}$ are plotted in fig 11,12,13 of Ref 11. Compared the calculated spectra in this paper with the measured, bremsstrahlung agree well with the measured spectra in mid-infrared, except the band below $2.5 \mu \mathrm{m}$, where the calculated spectrum is only one third of measured. Most lines from atom $\mathrm{N}$ and $\mathrm{O}$ agree well with the measured, but the calculated lines around $4 \mu \mathrm{m}$ are much less than measured, some important lines must be missed there. Because of the strong absorption from atmosphere around $2.7 \mu \mathrm{m}$, the measured spectrum from NO overtone is too small to analyze. There are some discrepancies for NO fundamental band. The radiation from vibrational band 1-0 is the same as measured, but when vibrational level rise, the measured spectrum gets larger than calculated. This result is easy to understand: HITEMP database is designed for temperature under $3000 \mathrm{~K}$, dealing with the plasma around $7500 \mathrm{~K}$ there must be some lines missing. Although we have done a lot of work to consider higher rovibrational transitions, but it is obvious not enough. We will go on the ab initio theory work in further.

\section{SAMPLE CALCULATIONS}

Two samples are calculated in this paper to analyze the mid-infrared spectra from hypersonic sphere. One sample is high pressure condition; the other sample is low pressure condition. The components taken into account for flow field are $\mathrm{N}_{2}, \mathrm{O}_{2}, \mathrm{NO}, \mathrm{N}, \mathrm{O}, \mathrm{N}^{+}, \mathrm{O}^{+}, \mathrm{NO}^{+}, \mathrm{N}_{2}^{+}, \mathrm{O}_{2}{ }^{+}, \mathrm{CO}_{2}, \mathrm{CO}_{\text {, }}$ $\mathrm{e}^{-}$. Among those components, the mid-infrared spectra from $\mathrm{N}, \mathrm{O}, \mathrm{NO}, \mathrm{CO}_{2}, \mathrm{CO}, \mathrm{e}^{-}$are calculated in this paper. 
Table 1 condition for tow samples

\begin{tabular}{|c|c|c|}
\hline parameters & Sample 1 & Sample 2 \\
\hline Temperature(K) & 300 & 245.2 \\
\hline Pressure(Pa) & 6000 & 652 \\
\hline Speed(km/s) & 5 & 5 \\
\hline Mole fraction & 0.808 & 0.81 \\
\hline N2 & 0.187 & 0.188 \\
\hline O2 & $2.15 \mathrm{e}-4$ & $2.15 \mathrm{e}-4$ \\
\hline $\mathrm{CO} 2$ & $2.54 \mathrm{e}-8$ & $3.74 \mathrm{e}-8$ \\
\hline $\mathrm{CO}$ & 0 & 0 \\
\hline $\mathrm{H} 2 \mathrm{O}$ & & 0 \\
\hline
\end{tabular}

\section{1 analyses of high pressure condition}

In such a dense atmosphere, the energy of flow field around hypersonic sphere can be very high, and it is thermodynamics equilibrium. The highest temperature in flow field around sphere is $10387 \mathrm{~K}$ near stagnation point. In such a high temperature flow field, nearly all $\mathrm{CO}_{2}$ molecules dissociate into $\mathrm{CO}$, a lot of $\mathrm{N}$ and $\mathrm{O}$ atoms dissociate from molecules, the mass fraction of $\mathrm{NO}$ can reach $17 \%$ in bow shock and $10 \%$ in wake.

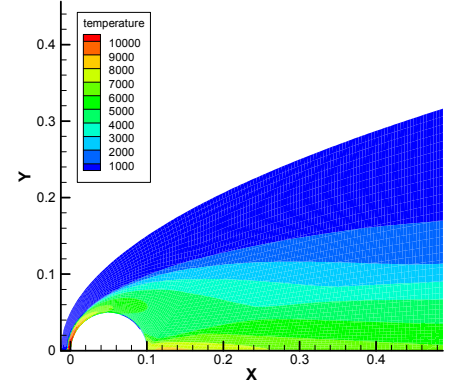

(a)

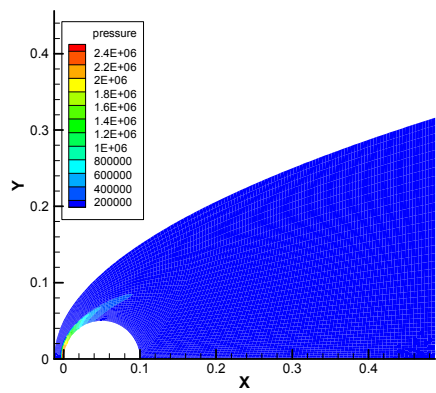

(b)

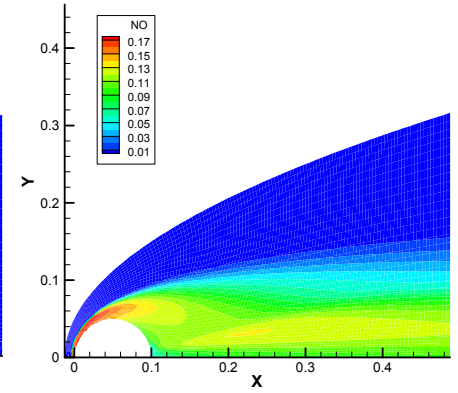

(c)

Figure 4 theory calculated contour for flow field around hypersonic sphere in high pressure condition

(a): temperature; (b): pressure; (c): mass fraction of $\mathrm{NO}$

According to the flow field result, the spectra of gas between $2 \mu \mathrm{m}$ and $6 \mu \mathrm{m}$ has been calculated without the radiation from sphere body itself. 


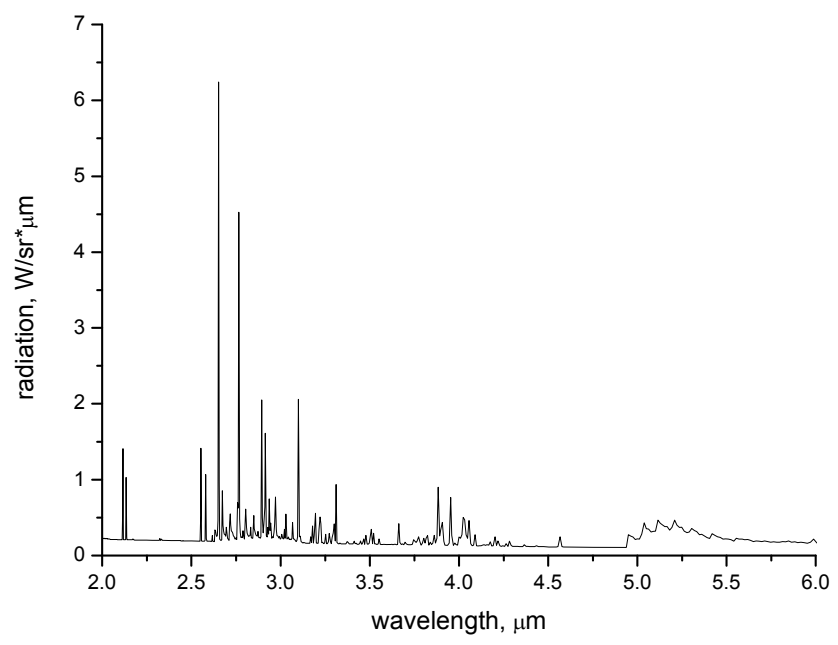

Figure 5 the spectra from hypersonic flow field in high pressure condition

As can be seen from the calculated spectra, vibrational-rotational bands of NO, lines of atom $\mathrm{N}$ and $\mathrm{O}$, bremsstrahlungs are the major sources of radiation. Because of the completely dissociation, there is no radiation from $\mathrm{CO}_{2}$. Although all $\mathrm{CO}_{2}$ transformed into $\mathrm{CO}$, the concentration of $\mathrm{CO}$ reach $2 \times 10^{-4}$, but band of CO is still too small, the total radiation of CO in $2-6 \mu \mathrm{m}$ is only $4 \times$ $10^{-4} \mathrm{~W} / \mathrm{sr}$. The radiations from different source are showed below.

Table 2 the radiation from different source

\begin{tabular}{|c|c|}
\hline source & Radiation, W/sr \\
\hline NO overtone band & 0.021 \\
\hline NO fundament band & 0.100 \\
\hline atom & 0.176 \\
\hline bremsstrahlung & 0.568 \\
\hline CO band & $4.3 \mathrm{e}-4$ \\
\hline Total & 0.972 \\
\hline
\end{tabular}

The infrared images are also calculated. In different wave range, the infrared images look very different. 


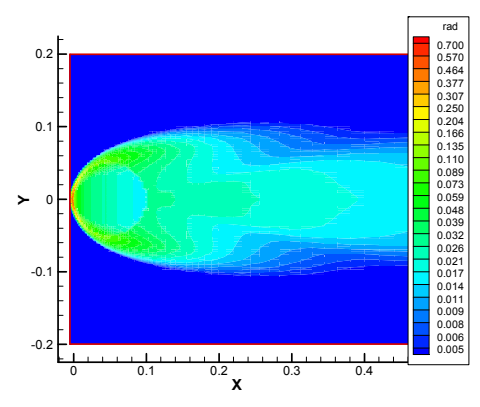

(a)

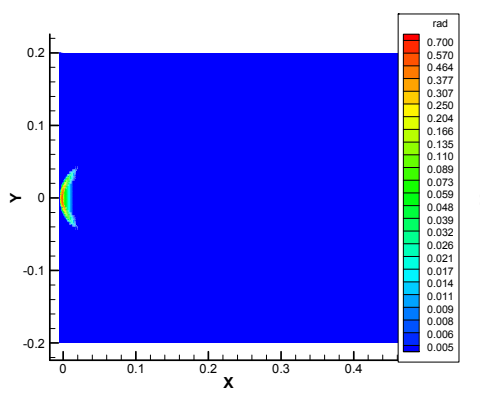

(b)

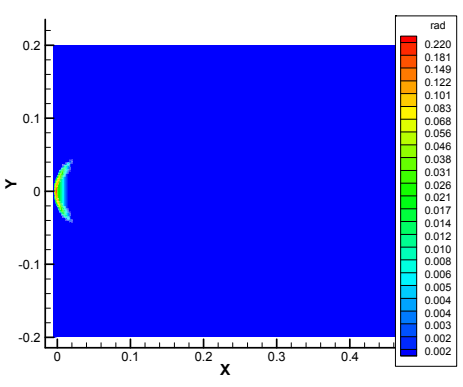

(c)

Figure 6 the infrared image of flow field around hypersonic sphere in different wave range

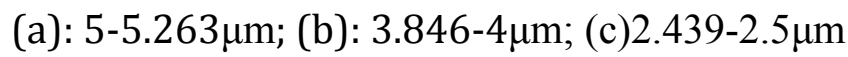

The NO fundament band can be excited in relatively low temperature, so it locates widely in flow field. The atoms locate mainly near stagnation point, and the atomic lines in infrared are almost transitions from very high energy near ionization which need very high temperature to be excited, so the atomic radiation is mainly from bow shock. The bremsstrahlung is dependent on electron density, it is very sensitive to temperature, so the bremsstrahlung is mainly located near stagnation point.

\section{2 analyses of low pressure condition}

In low pressure condition, the energy of flow field is much smaller than high pressure condition, and in some part of the flow field, it is in thermal nonequilibirium. The highest translational temperature in flow field is $7776 \mathrm{~K}$, and the highest vibrational temperature is $6692 \mathrm{~K}$. Even in low pressure condition, the dissociation of $\mathrm{CO}_{2}$ is also complete, but the concentrations of atoms, $\mathrm{NO}$ and electron are smaller than high pressure condition.

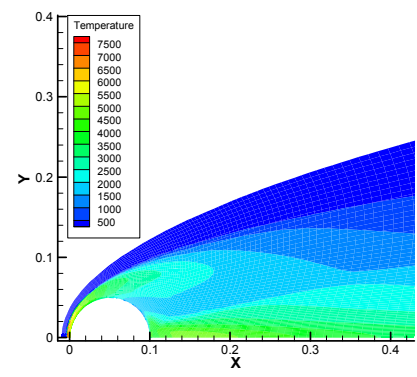

(a)

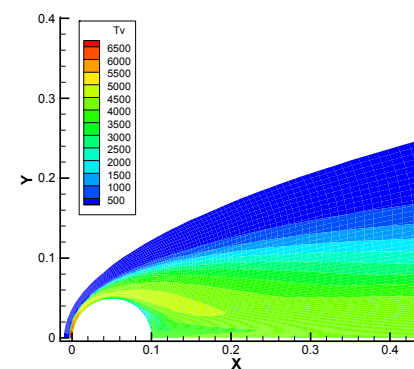

(b)

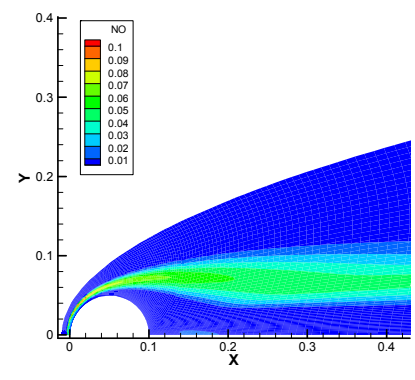

(c)

Figure 7 theory calculated contour for flow field around hypersonic sphere in low pressure condition 
(a): translational temperature; (b): vibratioanl temperature; (c): mass fraction of NO

In nonequilibirium condition, the simple two temperature model is used to solve radiation. And the calculated spectra is showed below.

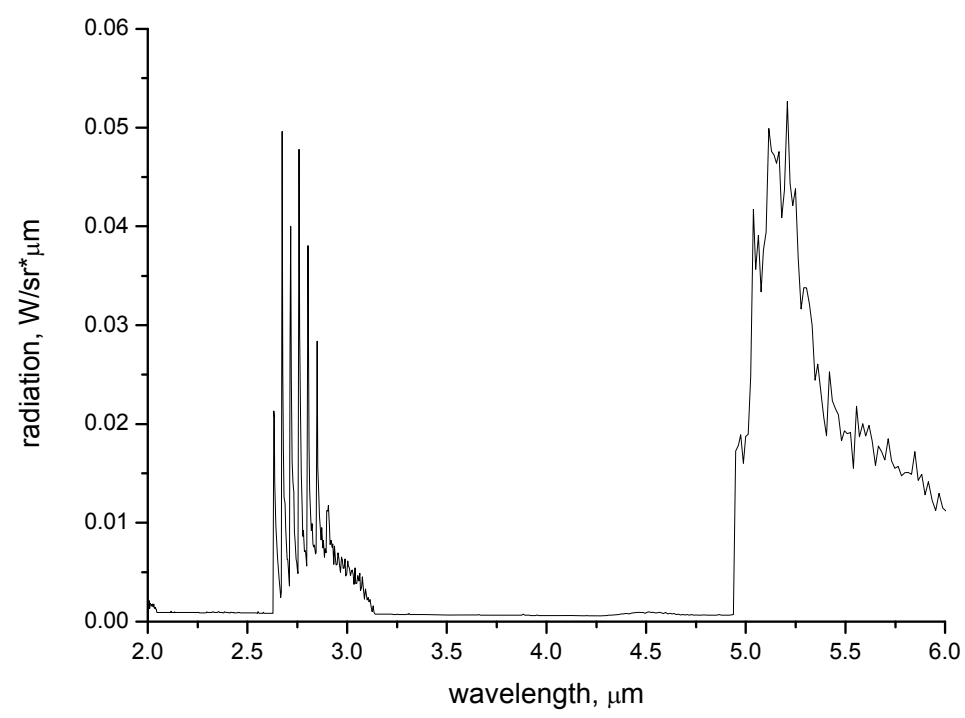

Figure 8 the spectra from hypersonic flow field in high pressure condition

For the low degree of dissociation and ionization, the radiation from atoms and bremsstrahlung is too small to be recognized. Nearly all the radiation is from NO fundamental and overtone bands.

The calculated infrared images show below:

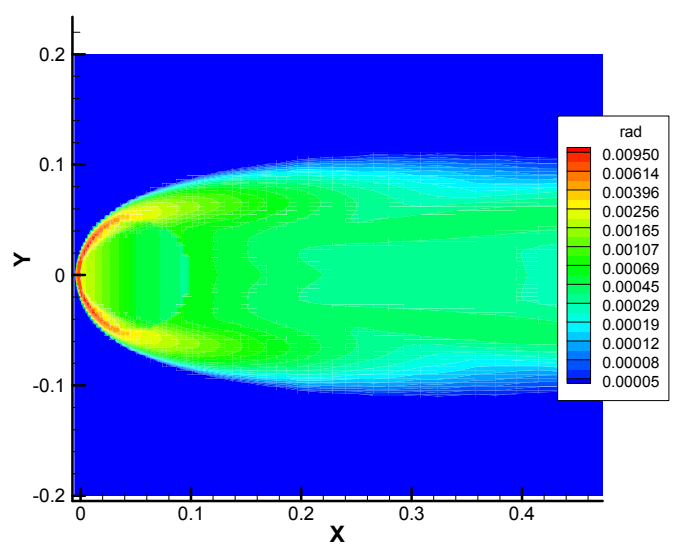

(a)

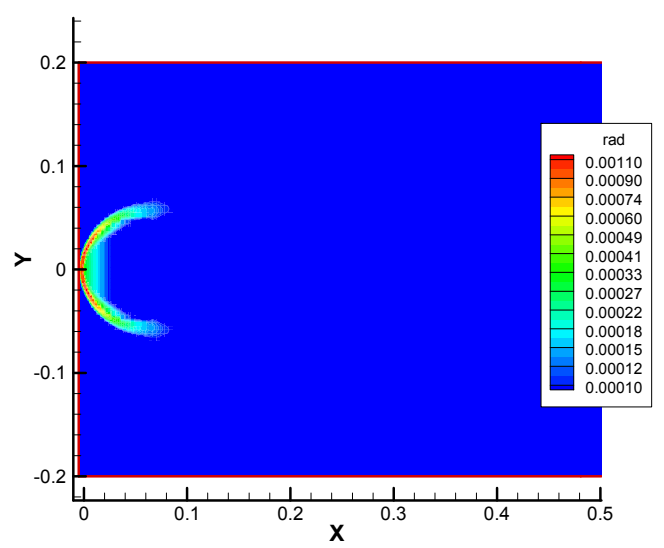

(b)

Figure 9 the infrared image of flow field around hypersonic sphere in different wave range (a): $5-5.263 \mu \mathrm{m} ;$ (b): $2.777-2.857 \mu \mathrm{m}$ 
The NO overtone band is only emitted from the head of bow shock, while the fundamental band is emitted from much wider area.

\section{CONCLUSIONS}

Pure dry air is not a strong infrared radiation source, because the main components of air such as $\mathrm{N}_{2}$ and $\mathrm{O}_{2}$ cannot emit infrared radiation. Although in high-temperature flow field, there are many chemical reactions, many new components produced, but according to our calculated result, only NO bands, atom radiation and bremsstrahlung are infrared radiation source. NO's two vibrational bands are strongest infrared source for flow field. The radiation from atoms and bremsstrahlung are dependent on high-temperature excitement, when density or speed turns down, they disappear quickly.

When a object's speed reaches $5 \mathrm{~km} / \mathrm{s}$, the temperature of the object's surface will be very high. Take the sphere in sample as an example, if the sphere is assumed as black body and surface temperature reaches $1000 \mathrm{~K}$, the spectra radiation will be $10-18 \mathrm{~W} / \mathrm{sr} / \mu \mathrm{m}$ in the mid-infrared; when

surface temperature reaches $2000 \mathrm{~K}$, the spectra radiation will be $50-800 \mathrm{~W} / \mathrm{sr} / \mu \mathrm{m}$. Compared to that radiation, the spectra radiation from hypersonic flow field is too small.

In the theory calculation, only simple two temperature model was used to deal with nonequilibirium condition. The error of this model remains unknown. Using a more precise model to analyze nonequilibirium radiation is the next step of our research.

\section{REFERENCES}

1. C. Park: NASA-TM-86707 (1985).

2. C. O. Laux, T. G. Spence, C. H. Kruger and R. N. Zare: Plasma Sources Science and Technology. 12(2003)125.

3. C. Park: Progress in Astronautics and Aeronautics. 96(1985)511.

4. R. C. Millikan, D. R. White:J. Chem. Phys. 39(1963) 3209.

5. M. F. Modest: Radiative Heat Transfer ( Academic Press, 2003) 2nd ed., p.326. 
6. L. S. Rothman, I. E. Gordon, R. J. Barber, H. Dothe, R. R. Gamache, A. Goldman, V. I. Perevalov, S. A. Tashkun, J. Tennyson : J. Quant. Spectrosc. Radiat. Transfer. 111(2010)2139.

7. H. J. Chen, X. L. Cheng, H. Liu, J. Wu: J. Quant. Spectrosc. Radiat. Transfer. 142(2014):49.

8. R. S. McDowell: J. chem. Phys. 93(1990) 2801.

9. J. O. Arnold, E. E. Whiting and G. C. Lyle: J. Quant. Spectrosc. Radiat. Transfer. 9(1969)775.

10. B. Kivel.: J. Quant. Spectrosc. Radiat. Transfer. 7(1967)51.

11. C. O. Laux, R. J. Gessman, B. Hilbert and C. H. Kruger: AIAA paper 95-2124 (1995).

12. A. Goldman, R. R. Gamache, A. Perrin, J. M. Flaud, C. P. Rinsland and L. S. Rothman: J. Quant. Spectrosc. Radiat. Transfer. 66(2000)455.

13. R. R. Gamache, S. Kennedy, R. Hawkins and L. S. Rothman: Journal of Molecular Structure. 517(2000) 407. 